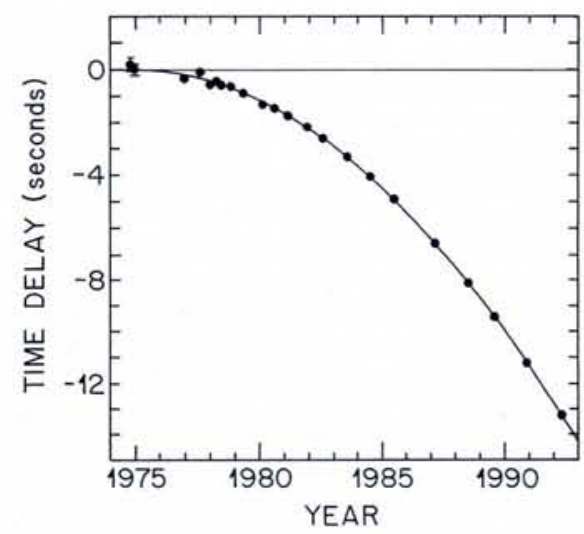

passes this " $\dot{\omega}-\gamma-\dot{P}_{b}$ " test perfectly at the present level of accuracy $(\approx 0.35 \%)$. It should be noted that the achieved accuracy is now so high that even a small correction for the effect of galactic acceleration needs to be included [12] (see Table 2 for details).

Within less than 10 years, two more binary pulsar systems, the one just mentioned (PSR B1534+12) and PSR B2127+11C, will presumably yield $\dot{\omega}-\gamma-\dot{P}_{\mathrm{b}}$ tests of gravitational radiation damping at the $1 \%$ level or
Fig. 3-Delay in the time of periastron of the binary pulsar PSR $1913+16$, relative to a non-dissipative model in which the orbital period remains fixed. Filled circles represent the measured shifts, while the smooth curve corresponds to the prediction of general relativity. (From [4].)

better [4]. In particular, PSR B1534+12 is a very interesting system because at least five post-Keplerian parameters can be accurately determined.

It should be clear from these brief remarks that the eight-hour binary pulsar PSR $1913+16$ has proven to be an outstanding laboratory for relativity. No relativist had ever dreamed of such a system before it was discovered. The high expectations held shortly after the discovery by Hulse and Taylor have been surpassed during the subsequent detailed study by Joe Taylor and his associates over almost 20 years. The work is an example of small science at its best and will survive as one of the most beautiful facets of physics in recent times. With it, general relativity has passed new, strong-field tests with complete success.
[1] Hulse R.A. \& Taylor J.H., Astrophys. J. 195 (1975) L51.

[2] Taylor J.H., Fowler A. and McCulloch P.M., Nature 277 (1979) 437; Taylor J.H. \& Weisberg J.M., Astrophys. J. 253 (1982) 908.

[3] ibid, 345 (1989) 434.

[4] Taylor J.H., Class. Quantum Gravity 1 (1993) 167.

[5] Blanford R. \& Teukolsky S.A., Astrophys. J. 205 (1976) 580; Epstein R., Astrophys. J. 216 (1977) 92 \& errata, 231 (1977) 644; Haugan M.P., Astrophys. J. 296 (1985) 1.

[6] Damour T. \& Deruelle N., Ann. Inst. H. Poincaré (Physique Théorique) 43 (1985) 107; ibid 44 (1986) 263.

[7] Damour T., 300 Years of Gravitation, Eds. S.W. Hawking \& W. Israel (Cambridge Univ. Press) 1982; idem., Proc. 2nd Canadian Conf. on General Relativity \& Relativistic Astrophysics, Eds.: A. Coley, C. Dyer \& T. Tupper (World Scientific Press) 1988.

[8] Damour T. \& Taylor J.H., Phys. Rev. D 45 (1992) 1840.

[9] Taylor J.H., Phil. Trans. R. Soc. A 341 (1992) 117.

[10] Wolszczan A., Nature 350 (1991) 688.

[11] Taylor J.H. et al., Nature 355 (1992) 132.

[12] Damour T. \& Taylor J.H., Astrophys. J. 366 (1991) 501.

\title{
ACTIVE GALACTIC NUCLEI
}

\section{Extreme UV Now the Issue}

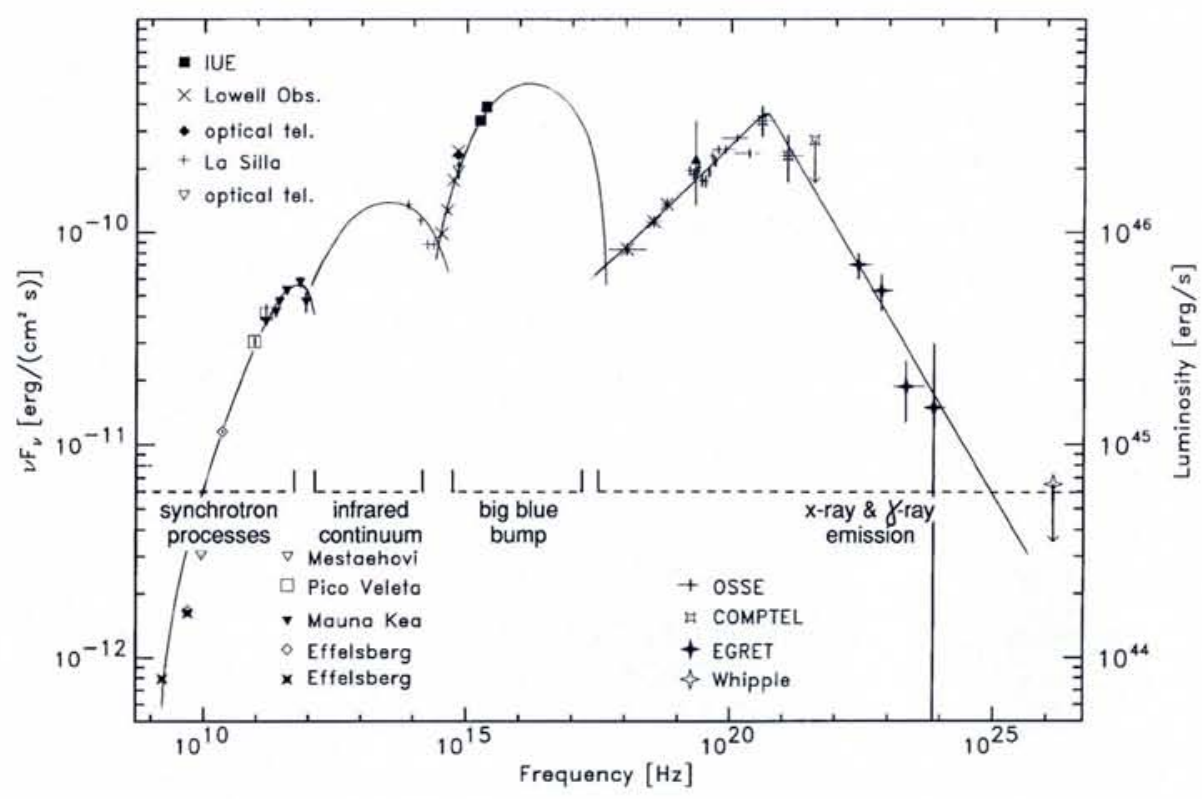

Active galactic nuclei (AGN) represent the brightest objects in the Universe. Energy emitted by AGN is distributed across the whole electromagnetic spectrum owing to different physical processes that are far from understood (Fig. 1). As variations of the energy flux are observed in all spectral bands on relatively short time-scales, the main goals of several worldwide observational programmes currently underway are to measure the multi-wavelength continua of these sources with Earth- and space-based observatories, to map the variations, and to uncover correlations between the different emission components.
The International Astronomical Union's (IAU) 159th symposium entitled Active Galactic Nuclei across the Electromagnetic Spectrum (August 30 - September 3, 1993) organized by Geneva Observatory reviewed recent progress. Attended by 300 participants from 31 countries with 200 posters and 50 oral contributions, there were reports on theory and observations on AGN involving data spanning 15 decades of the electromagnetic spectrum. Participants were reminded by $L$. Woltjer (the President of the European Astronomical Society) that the occasion marked the 30th anniversary of the discovery of quasars that lie at the cores of
Fig. 1 - Energy-density spectrum of the Seyfert Type 1 quasar 3C 273 from quasisimultaneous observations obtained with ground-based observatories and with the satellites IUE, Ginga and CGRO [Lichti et al., this symposium]. This hitherto unpublished energy spectrum represents the first time that simultaneous observations spanning such a large frequency range have been combined. It can be divided into four main components each originating from different physical processes.

extremely active galaxies, and the 50th anniversary of Seyfert's discovery of a particular class of active galaxies.

Concerning the main topic of the symposium, namely multi-wavelength continuum emission of $A G N$ and its variability, two main groups of $A G N$ are now recognized. Recalling that models of AGN generally involve massive black holes and central jets surrounded by accretion disks threaded by magnetic fields (see cover illustration), in the socalled blazar class, the featureless emission can be understood using models involving shocks in the jets. In Seyfert-type objects, including both radio-quiet and radio-loud sources, emission is dominated by several components (Fig. 1) which are either undetectable or not present in the former class. While the synchrotron origin of the radio emission and the contribution of reprocessing by dust to the infrared continua are now widely accepted, the origins of the optical to soft x-ray component and of the hard $x$-ray to $\gamma$-ray continuum remain controversial.

\section{Hard X-Ray to $\gamma$-Ray Range}

Our knowledge of the hard $\mathrm{x}$-ray and $\gamma$-ray emission of AGN has increased rapidly in the last five years with the results, reviewed during the meeting, of the Ginga, Granat and Compton GRO satellites and of the groundbased Whipple TeV-observatory.

In a fraction of the active nuclei, which are thought to be observed perpendicular to the accretion disc (i.e., along the jet), the $\gamma$-rays 
result from the interaction of relativistic particles moving in the jet with soft photons. One reasonable approach considers the enhancement by relativistic electrons of soft photons coming from an accretion disk and scattered in the direction of the jet. Such models are able to produce $\gamma$-rays with a flat spectrum $\left(\mathrm{F}_{\nu} \sim \nu^{-1}\right)$ up to energies of $1 \mathrm{TeV}$. For objects behaving in this way, the apparent energy flux released in the $\gamma$-rays can be 10 to 100 times larger than the fluxes observed in the other energy bands.

The hard $x$-ray spectra of the few Seyferttype objects that have been monitored in the soft $x$-ray range seem to cut off between 100 $\mathrm{keV}$ and $10 \mathrm{MeV}$. This spectral feature is interpreted using either non-thermal models involving pair cascades and absorption or quasi-thermal clouds of pairs or electrons. Repeated observations at improved spectral resolution and sensitivity are needed to determine which of these interpretations is correct.

\section{Optical to Soft X-Ray Range}

The origin of the optical to soft $\mathrm{x}$-ray emission is another key point in analyzing the AGN spectra. Until five years ago, this wavelength range was interpreted in terms of direct emission from the accreting matter Several multi-wavelength studies (reviewed or presented during the symposium and involving ground-based observatories and data from the IUE, Hubble Space Telescope and ROSAT satellites) have shown that the situation is much more complicated. Both the observed variations and spectral shapes are not compatible with simple accretion disks. The main problem is that both the timescales of variability and the lags observed between variations in different energy bands are several orders of magnitude too small, when compared to the size of the emitting region and to the expected sound velocity. Under investigation are various models involving energetic photons reprocessed by either optically thick matter (accretion disk) or optically thin, dense cloudlets.

Although galactic absorption makes observations in the extreme ultraviolet (UV) very difficult, first results from NASA's Extreme Ultraviolet Explorer launched in 1992 were presented at the meeting. The number of AGN detected so far is consistent with the presence of excess flux in the extreme UV. Emission-line diagnostics at soft $\mathrm{x}$-rays will be a key to understanding the state of the reprocessing matter. The first medium-resolution $(E / \Delta E=50$, where $E$ is the energy) $x$-ray spectra optimised for iron emission of AGN have been obtained with the Japanese satellite ASCA launched last February with the largest high-resolution CCD array ever used in space. ASCA represents the wave of the future and the European Space Agency's INTEGRAL $\gamma$-ray observatory selected in June for the next Medium-Sized Mission incorporates the same approach. ASCA's preliminary spectra, presented for the first time to the international community at the meeting, suggest that apart from the iron complex at $6.5 \mathrm{keV}$, the number of resolved lines is smaller than expected for some models of reprocessing.

\section{Unified Models}

The last session of the symposium dealt with unified models of AGN. Optical polariza-

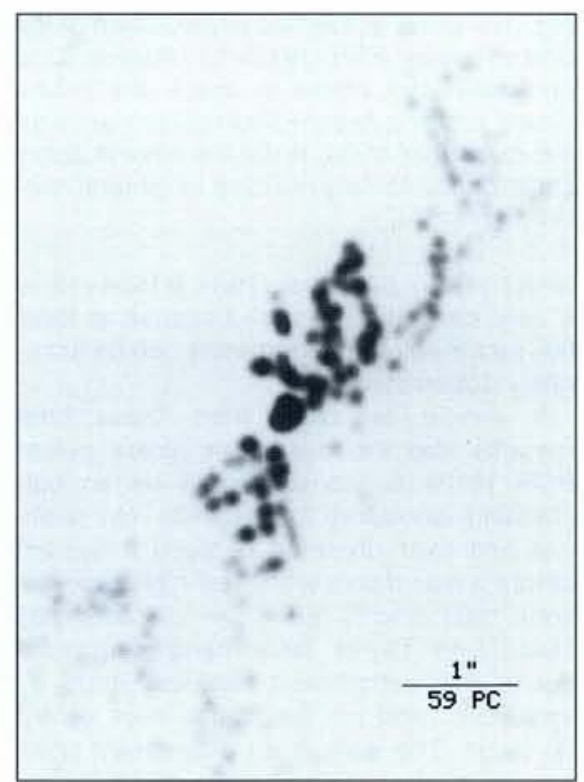

tion studies confirm the view that the active nucleus is heavily absorbed in part of the AGN (Seyfert 2), and that the absorbing matter is probably distributed in a torus geometry. Thus, unified models have the advantage of being able to explain the existence of different kinds of AGN (Seyfert 1 and 2) at different viewing angles. Images (e.g., Fig. 2) obtained with the Hubble Space Telescope (HST) of narrow emission line clouds photo-ionized by the central, obscured nucleus in a bi-conical geometry confirm this general picture, but show that the obscuring torus may be patchy or less optically thick in some directions. Inconsistencies with the simple unified model are also derived from mapping of the infrared emission at high spatial resolution by ground-based observatories, revealing that the absorbing matter is not distributed in a simple torus, and from ROSAT and IUE observations uncovering intermediate objects between Seyfert Type 1 and Type 2

While the basic idea behind unified models is probably valid, the geometry of the inner 100 parsec cannot be considered as unique
Fig. $2-A$ reconstruction of the continuumsubtracted [O III] $\lambda 5007 \AA$ image of the Seyfert Type 1 galaxy NGC 4151 obtained with the Planetry Camera aboard the Hubble Space Telescope at a resolution of 0.085 " [Z. Tsvetanov et al., this symposium]. Each dark patch represents a region of strong emission in the $[0 \mathrm{III}]$ line. A bi-conically shaped extended emission is clearly observed. In the frame of unified models of AGN, this geometry is explained by the fact that the central photo-ionizing source is obscured by a thick torus of gas and dust. Only the clouds located perpendicularly to this torus are photo-ionized and emit lines. It should be noted that the emission line cones support a much larger angle than the jets shown in the cover illustration. While several similar images have been reported recently, the HST image is the first to provide the main geometrical parameters of the source. In this particular case, the line of sight passes through the absorbing torus so unified models predict an absorbed nucleus. However, the nucleus of the source is observed most of the time without any sign of absorption in the optical and UV bands (but shows absorption in the $x$-rays).

in all active galaxies. At the last IAU symposium on the subject five years ago, the lack of knowledge on the multi-waveband spectra of AGN made very likely the theoretical suggestion that a bump in the UV range of the spectrum corresponded to direct emission by the accreting matter. Today, the origin of the extreme UV emission has become the most important unresolved question, given that the electromagnetic spectrum is completely observable with the exception of very energetic $\gamma$-rays and with serious limitations for the very energetic and extreme UV domains.

\section{Roland Walter}

Max Plank Institut für extraterrestrische Physik Garching bei München

\section{Niels Bohr Institute FELLOWSHIPS}

The email address for correspondence quoted in the October 1993 issue should read "uholm@nbivax.nbi.dk".

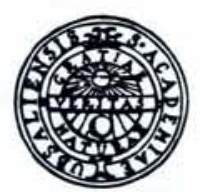

\section{UPPSALA UNIVERSITET \\ SENIOR LECTURER IN THEORETICAL ELECTROTECHNICS, ESPECIALLY PLASMA PHYSICS}

Ref $\mathrm{nr} 6902 / 93$

Required qualifications are a Ph.D. degree, pedagogical proficiency and good written and spoken commands of Swedish or English.

Special importance will be attached to scientific and teaching proficiency, in equal degrees. "Scientific proficiency" refers to the applicant's own research, particularly theoretical research on magnetically confined plasmas. The successful applicant is expected to contribute to research within the Euratom fusion programme. "Teaching proficiency" refers to teaching, supervision, planning and direction of teaching and compilation of teaching materials.

The applications should include a curriculum vitae, copies of degree certificates, an account of previous research achievements and teaching merits, a list of publications and reprints according to the list. The documents should be submitted in two copies.

The university is an equal opportunity employer.

The application should be directed to the Vice Chancellor, Uppsala University, P.O. Box 256, S-751 05 Uppsala, Sweden. Closing date: 15 December 1993.

Detailed information and formal rules for application will be given by the head of the Department of Technology, Professsor Mats Olsmats, P.O. Box 534, S-751 21 Uppsala, Sweden. Telephone: +46 (18) 1830 72; Fax: +46 (18) 555095. 